\title{
Multistate Complete-Active-Space Second-Order Perturbation Theory Based on Density Matrix Renormalization Group Reference States
}

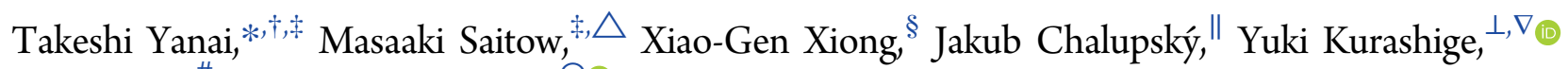
Sheng Guo," and Sandeep Sharma ${ }^{\circ}$

${ }^{\dagger}$ Department of Theoretical and Computational Molecular Science, Institute for Molecular Science, Okazaki, 444-8585 Aichi Japan

The Graduate University for Advanced Studies, Myodaiji, Okazaki, Aichi 444-8585, Japan

${ }^{\S}$ Shanghai Institute of Applied Physics, Chinese Academy of Sciences, Shanghai 201800, China

"Institute of Organic Chemistry and Biochemistry, Academy of Sciences of the Czech Republic, Flemingovo nám. 2, 16110 Prague 6, Czech Republic

${ }^{\perp}$ Department of Chemistry, Graduate School of Science, Kyoto University, Kitashirakawa-Oiwake-cho, Sakyou-ku, Kyoto 606-8520, Japan

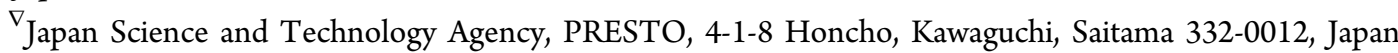

"Division of Chemistry and Chemical Engineering, California Institute of Technology, Pasadena, California 91125, United States

${ }^{\circ}$ Department of Chemistry and Biochemistry, University of Colorado, Boulder, Boulder, Colorado 80302, United States

Supporting Information

ABSTRACT: We present the development of the multistate multireference second-order perturbation theory (CASPT2) with multiroot references, which are described using the density matrix renormalization group (DMRG) method to handle a large active space. The multistate first-order wave functions are expanded into the internally contracted (IC) basis of the single-state single-reference (SS-SR) scheme, which is shown to be the most feasible variant to use DMRG references. The
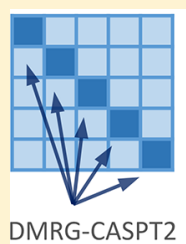

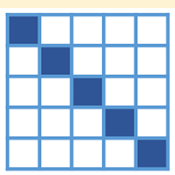

Multistate DMRG-CASPT2

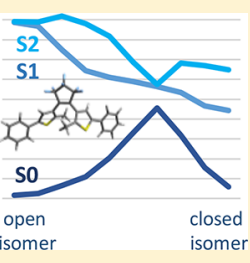
feasibility of the SS-SR scheme comes from two factors: first, it formally does not require the fourth-order transition reduced density matrix (TRDM) and second, the computational complexity scales linearly with the number of the reference states. The extended multistate (XMS) treatment is further incorporated, giving suited treatment of the zeroth-order Hamiltonian despite the fact that the SS-SR based IC basis is not invariant with respect to the XMS rotation. In addition, the state-specific fourthorder reduced density matrix (RDM) is eliminated in an approximate fashion using the cumulant reconstruction formula, as also done in the previous state-specific DMRG-cu(4)-CASPT2 approach. The resultant method, referred to as DMRG-cu(4)-XMSCASPT2, uses the RDMs and TRDMs of up to third-order provided by the DMRG calculation. The multistate potential energy curves of the photoisomerization of diarylethene derivatives with CAS $(26 \mathrm{e}, 24 \mathrm{o})$ are presented to illustrate the applicability of our theoretical approach.

\section{INTRODUCTION}

Calculation of electronically excited states is challenging because they often involve a strong multireference (MR) electronic character. ${ }^{1,2}$ It requires a model to efficiently and accurately describe a highly correlated configuration expansion of the many-electron wave functions. Various frameworks of MR theory for the excited-state calculation have been established and widely used, such as the MR configuration interaction (MRCI) method, ${ }^{3-5}$ complete active space secondorder perturbation theory (CASPT2), ${ }^{6-9} \mathrm{MR}$ second-order perturbation theory (MRMP), ${ }^{10}$ multiconfigurational quasidegenerate perturbation theory (MCQDPT), ${ }^{11,12}$ N-electron valence state perturbation theory (NEVPT2), ${ }^{13,14}$ multireference linearized coupled-cluster theory (MRLCC), ${ }^{15-18}$ etc.
The CASPT2 theory is one of the most popular MR methods because of its low computational cost and theoretical simplicity. $^{6-9,19-27}$ In the CASPT2 calculations, the target excited states are first characterized in a qualitative manner by the complete active space self-consistent field (CASSCF) wave functions. ${ }^{28,29}$ They are used as the reference states accounting for the multiconfigurational nature of electron correlation, often referred to as static correlation. The state-averaged (SA) CASSCF treatment is usually used to obtain the references as a multiroot solution that provides a ground state and several lowlying excited states. Using these CASSCF states as the zerothorder, the CASPT2 method describes the first-order perturbed

Received: July 10, 2017

Published: September 7, 2017 
wave functions and second-order perturbation energies to further account for dynamic correlation. In practice, these perturbative corrections to the CASSCF references play an important role in obtaining a quantitative accuracy. For example, the relative energy levels of the CASSCF states are often corrected by the CASPT2 treatment.

There are two approaches based on CASPT2 to excited-state calculations: state-specific and multistate (MS).,11,12 In the state-specific CASPT2 theory, the perturbation corrections are separately evaluated for each CASSCF reference wave function. It is, however, well-known that the state-specific treatment is inadequate for studying near-degenerate states, in which the strong mixing arises between the reference and other secondary CASSCF states via the second-order interactions. This mixing can be properly accounted for by the MS-CASPT2 theory, ${ }^{8}$ an extension of CASPT2 based on the quasi-degenerate perturbation theory (QDPT) ${ }^{30-36}$ in conjunction with the multipartitioning technique. ${ }^{37,38}$ It constructs an effective Hamiltonian matrix in the reference state basis under the presence of the perturbation, and its diagonalization yields the mixing of the perturbed states. The MS extension of MR perturbation theory was similarly developed in the MCQDPT $^{11,12}$ and QD-NEVPT2 ${ }^{14}$ methods. The MSCASPT2 method was combined with Granovsky's extended MS (XMS) method ${ }^{39}$ by Shiozaki et al. to improve the zerothorder description by additionally accounting for the offdiagonal zeroth-order coupling. ${ }^{21}$

In recent years, the density matrix renormalization group (DMRG) theory of White ${ }^{40,41}$ has received great interest in the quantum chemistry community because it has a marked capability of efficiently treating a larger active space than was ever possible. ${ }^{42-61}$ It can be used as an exceedingly scalable alternative to the configuration interaction (CI) solver. The superior scalability of the DMRG method is owing to its efficient, specialized parametrization of quantum entanglement. ${ }^{62-65}$ In our previous studies, we presented an extension of the state-specific CASPT2 method using the CASSCF reference states that are described by the DMRG method. ${ }^{66,67}$ The incorporation of the DMRG solver into CASSCF and CASPT2 is referred to as DMRG-CASSCF ${ }^{54,68-72}$ and DMRGCASPT2, ${ }^{66,67,73}$ respectively. The applications of the DMRGbased state-specific CASPT2 method to molecular excited states were reported in refs 67 and 74-76.

A major complexity in the DMRG-CASPT2 lies in the fact that the CASPT2 theory requires the fourth-order reduced density matrix (4-RDM) of the reference wave function. The steep dependence of the computational cost and memory requirement of 4-RDM on the number of active orbitals hinders the use of the large active space for the conventional CASPT2 formalism. In our previous work, we introduced the use of the approximated 4-RDM that is obtained using the lower-particle rank RDMs via the cumulant reconstruction. ${ }^{77,78}$ The elimination of the pure 4-RDM is referred to as the $\mathrm{cu}(4)$ approximation and enables a large active space to be used in state-specific CASPT2 calculations. ${ }^{56,67,73}$ Its combination with the NEVPT2 method was studied in ref 79. The $\mathrm{cu}(4)$ approximation was also employed in the development of the DMRG-based MRCI method. ${ }^{59,80}$ The cumulant approximations to 3-RDM and third-order many-particle operators were investigated in the canonical transformation theory. ${ }^{81}$ Note that, in the initial DMRG-CASPT2 implementation shown in ref 66 , the approach to evaluate parts of the pure 4-RDM of the DMRG wave function was implemented in conjunction with the diagonal Fock treatment of the CASPT2-D approach. ${ }^{6}$ The efficient algorithm to compute RDMs from DMRG wave functions was developed by Zgid et al. ${ }^{82}$ and Chan et al. ${ }^{68} \mathrm{We}$ then presented its extension to the computation of $3-\mathrm{RDM}^{67}$ Sharma and Chan extended the 2-RDM algorithm for the spinadapted DMRG wave functions. ${ }^{83}$ The developments of the DMRG algorithms to compute the spin-adapted $k$-RDM and $k$ TRDM in the DMRG program code $\mathrm{BLOCK}^{84}$ are described in refs $85-87$. The applicability of the DMRG-CASPT2 method has been recently examined by the other groups. ${ }^{55,56,87}$

The dependence of the CASPT theory on the 4-RDM is associated with its formalism based on the internally contracted (IC) basis scheme. ${ }^{88}$ Let us here point out several alternative approaches which can avoid the $\mathrm{cu}(4)$ approximation for the dynamic correlation calculations with the DMRG reference states. Sharma and Chan showed the matrix product state (MPS) based PT formulation in which the first-order wave function of the Hylleraas functional is expressed with MPS in a similar manner to the uncontracted MR basis scheme free from the 4-RDM dependence. ${ }^{89}$ It was extended to a partially contracted MRPT scheme ${ }^{17}$ and the MPS based implementation of the MRLCC. ${ }^{18}$ Wouters et al. presented a formula to compute the pure 4-RDM used in DMRG-CASPT2 by exploiting the symmetry of the generalized Fock matrix ${ }^{55}$ and introducing a compressed MPS form of auxiliary excited configurations. Sokolov et al. developed a time-dependent formulation of the NEVPT2 theory, ${ }^{90}$ which bypasses the need for the IC formulation and thus does not require 4-RDM, and solve it by propagating the MPS. ${ }^{91}$ The work additionally presented the low-scaling MPS-based implementation of strongly contracted (sc) NEVPT2 using the variational compression techique. The combinations of the state-specific NEVPT2 and DMRG with the 4-RDM computation were investigated by Guo et al. using the descreased- $M$ MPS $^{85}$ and by Freitag et al. ${ }^{51}$ Roemelt et al. showed that the complexity of sc-NEVPT 2 was reduced by projecting the perturber functions as well as the unperturbed Hamiltonian onto a DMRG-based reduced Hilbert space. ${ }^{92}$

In this paper, we present the MS extension of the DMRGCASPT2 method with the $\mathrm{cu}(4)$ approximation to treat neardegenerate electronic states. Our approach is essentially based on the formalism of the MS-CASPT2 theory. It is mainly aimed at further describing the perturbation correction in the offdiagonal elements of the MS-CASPT2's effective Hamiltonian matrix. Sharma et al. developed the quasi-degenerate perturbation theory with DMRG references in a very similar spirit to the current study. ${ }^{93}$ It is based on the QD-NEVPT2 formalism modified to retain a model space invariance and on the MPS-PT to express the first-order correction directly derived from the MPS. ${ }^{17,89}$

In the following section, the brief overview of the (X)MSCASPT2 theory is provided to show the ansatz of the firstorder wave functions relative to the multiroot zeroth-order references, the working equations, and the effective Hamiltonian used in this work, followed by the detailed description of our approach to incorporate the XMS-CASPT2 theory into the DMRG reference states. Section 3 shows benchmark applications to assess accuracy and applicability. The concluding remarks are described in section 4. In the Appendices, the complementary techniques to regulate artificial intruder states caused by the $\mathrm{cu}(4)$ approximation and to reduce the storage of the first-order expansion coefficients partially using the diagonal approximation are provided; they 
are introduced to enhance the applicability of the present method.

\section{METHOD}

2.1. Overview of (X)MS-CASPT2 Theory. Here let us briefly review the extension of CASPT2 to the multistate (MS) calculation. The notation for indices is summarized in Table 1 .

\section{Table 1. Notation of Indices}

$\begin{array}{ll}L, M, N, P & \text { reference CAS-CI/SCF states or their rotated states } \\ & \left(=1, \ldots, n_{\text {state }}\right) \\ p, q, r, s & \text { generic orbitals } \\ v, w, x, y & \text { core (inactive) orbitals } \\ g, h, i, j, k, l, m, n & \text { active orbitals } \\ a, b, c, d & \text { external (virtual) orbitals } \\ \Omega, \Pi & \text { sets of four orbital indices for double-excitation } \\ & \text { operators }\end{array}$

Given a set of the reference states $\{|N\rangle\}$ Granovsky's extended MS (XMS) method introduces their rotation, written as $|\tilde{M}\rangle=$ $\sum_{N}|N\rangle U_{N M} \cdot{ }^{21,39}$ The rotated states are hereafter denoted by a tilde. Let the Fock operator be defined as $\hat{f}=\sum_{p q} f_{p q} \hat{E}_{p q}$ where $\hat{E}_{p q}$ is the spin-summed single excitation operator and $f_{p q}$ is a generalized Fock matrix based on the state-averaged 1-RDM. The Fock matrix in the state basis $\{|N\rangle\}$ is introduced as $f_{M N}=$ $\langle M|\hat{f}| N\rangle$, which is evaulated as $f_{M N}=\sum_{p q} f_{p q} D_{q}^{p}(M, N)$ with $D_{q}^{p}(M, N)=\left\langle M\left|\hat{E}_{p q}\right| N\right\rangle$ for 1-RDM $(\mathrm{M}=N)$ and 1-TRDM $(M$ $\neq N)$. The off-diagonal elements of $f_{M N}$ are in general nonzero. The rotation matrix $\mathbf{U}$ is obtained by diagonalizing $f_{M N}$ such that $\mathbf{U}^{\dagger} \mathbf{f U}=\tilde{\mathbf{f}}$ where $\tilde{\mathbf{f}}$ is a diagonal matrix written as $f_{M N}=\langle\tilde{M}|\hat{f}|$ $\tilde{N}\rangle=\delta_{M N} E_{M}^{(0)}$. The diagonal element $E_{M}^{(0)}$ corresponds to the zeroth-order energy of the rotated reference state. The stateaveraged Fock operator $\hat{f}$ is assumed to be constructed from an equally weighted ensemble of the first-order reduced density matrices (1-RDMs) of all of the references, so that it is invariant with respect to the XMS rotation. The original MS formalism of MS-CASPT2 ${ }^{8}$ does not consider this rotation and is thus equivalent to the use of the identity matrix for $\mathbf{U}$.

In the main framework of CASPT2, the first-order wave function is expanded into the internally contracted (IC) basis, ${ }^{88}$ which are generated by applying excitation operators to the zeroth-order reference. There are two variants of the IC basis scheme for (X)MS-CASPT2, referred to as the multistate multireference (MS-MR) and single-state single-reference (SSSR) contractions, respectively. ${ }^{5}$ In the MS-MR scheme, the union of the IC basis generated from all the reference CAS-CI/ SCF states is commonly used for representing the perturbed wave functions of all of the states. When incorporated into XMS-CASPT2 theory, it can retain the invariance of the theory with respect to the unitary mixing of the references. The SS-SR contraction is essentially the same as the IC basis of the statespecific CASPT2 theory, in which the IC configurations are generated solely relative to a single CAS-CI/SCF state of the target. The IC basis of the MS-MR scheme is thus complete in the sense that they consistently span the first-order space of all of the states, but that of the SS-SR scheme spans its subspace that varies depending on the target state, as shown in Figure 1.

The procedure to obtain the first-order wave function in the (X)MS-CASPT2 theory is 2-fold. First, the state-specific firstorder wave functions $\left\{\Psi_{L}^{(1)}\right\}$, i.e., the perturbation to each reference $|\tilde{L}\rangle$, are determined. They are parametrized in the MS-MR scheme as

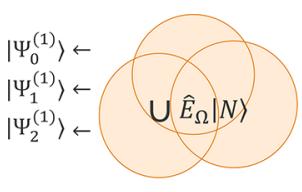

(a) MS-MR

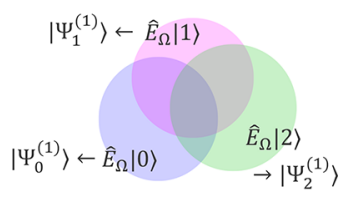

(b) SS-SR

Figure 1. Schematic illustration of the first-order interaction spaces spanned by the IC basis of the (a) MS-MR and (b) SS-SR schemes of the (X)MS-CASPT2 theory, whose expansions are given in eqs 1 and 2 , respectively.

$$
\left|\Psi_{L}^{(1)}\right\rangle=\sum_{N} \sum_{\Omega} \hat{E}_{\Omega}|\tilde{N}\rangle T_{\Omega, L N}, \quad(\mathrm{MS}-\mathrm{MR})
$$

where $\hat{E}_{\Omega}$ are the spin-summed double excitation operators $\left(\left\{\hat{E}_{\Omega}\right\}=\left\{\hat{E}_{p q} \hat{E}_{r s}\right\}\right)$, the detailed definitions of which can be consulted in refs 66 and 67 . The SS-SR scheme is based on the following ansatz:

$$
\left|\Psi_{L}^{(1)}\right\rangle=\sum_{\Omega} \hat{E}_{\Omega}|\tilde{L}\rangle T_{\Omega, L L}, \quad(\mathrm{SS}-\mathrm{SR})
$$

which can be viewed as spanning a subspace of the expansion of eq 1. The coefficients, $\left\{T_{\Omega, L N}\right\}$ (eq 1 ) or $\left\{T_{\Omega, L L}\right\}$ (eq 2), are determined by the following amplitude equations, which are solved separately for each target reference $|\tilde{L}\rangle$

$$
\left\langle\tilde{M}\left|\hat{E}_{\Omega}^{\dagger}\left(\hat{f}-E_{L}^{(0)}\right)\right| \Psi_{L}^{(1)}\right\rangle+\left\langle\tilde{M}\left|\hat{E}_{\Omega}^{\dagger} \hat{H}\right| \tilde{L}\right\rangle=0
$$

with $\forall M \in\left\{1, \ldots, n_{\text {state }}\right\}$ for MS-MR and with $M$ set to the given $L$ for SS-SR.

Second, we proceed to the construction of the effective subspace Hamiltonian

$$
H_{M L}^{\mathrm{eff}}=H_{M L}^{\mathrm{ref}}+\frac{1}{2}\left(H_{M L}^{(2)}+H_{L M}^{(2)}\right)
$$

where the Hamiltonian matrix to the first-order is given by $H_{M L}^{\text {ref }}$ $=\langle\tilde{M}|\hat{H}| \tilde{L}\rangle$, and the dynamic correlation arising between the perturbed states is determined by the second-order perturbation

$$
H_{M L}^{(2)}=\left\langle\tilde{M}|\hat{H}| \Psi_{L}^{(1)}\right\rangle
$$

The eigenvalues resulting from the diagonalization of $\mathbf{H}^{\text {eff }}$ are the XMS-CASPT2 energies $E_{P}^{\mathrm{MS}}$, and the associated wave functions $\left|\Psi_{P}^{\mathrm{MS}}\right\rangle$ are obtained using the eigenvectors $R_{L P}$ :

$$
\begin{aligned}
& E_{P}^{\mathrm{MS}}=\sum_{M L} H_{M L}^{\mathrm{eff}} R_{M P} R_{L P} \\
& \left|\Psi_{P}^{\mathrm{MS}}\right\rangle=\sum_{L}\left(|\tilde{L}\rangle+\left|\Psi_{L}^{(1)}\right\rangle\right) R_{L P}
\end{aligned}
$$

Let the vector $\boldsymbol{\sigma}^{(L, M, N)}$ be defined as

$$
\begin{aligned}
\sigma_{\Omega}^{(L, M, N)}= & \sum_{\Pi}\left[\left\langle\tilde{M}\left|\hat{E}_{\Omega}^{\dagger}\left(\hat{f}-E_{L}^{(0)}\right) \hat{E}_{\Pi}\right| \tilde{N}\right\rangle T_{\Pi, L N}\right] \\
& +\delta_{L N}\left\langle\tilde{M}\left|\hat{E}_{\Omega}^{\dagger} \hat{H}\right| \tilde{L}\right\rangle
\end{aligned}
$$


which is a core part of the computation of the amplitude equation (eq 3 ). Note that, for a given $(L, M, N)$, the complexity of computing $\boldsymbol{\sigma}^{(L, M, N)}$ is independent of the number of the reference states (i.e., $n_{\text {state }}$ ). Equation 3 can be rewritten using eq 8 for the MS-MR and SS-SR schemes as

$$
\sum_{N} \boldsymbol{\sigma}^{(L, M, N)}=\mathbf{0}, \forall L, \forall M, \quad(\mathrm{MS}-\mathrm{MR})
$$

and

$$
\boldsymbol{\sigma}^{(L, L, L)}=\mathbf{0}, \forall L, \quad(S S-S R)
$$

respectively. These expressions indicate that, for a given system, the scaling of computational cost of XMS-CASPT 2 with $n_{\text {state }}$ is cubic $\left(O\left(n_{\text {state }}^{3}\right)\right)$ for MS-MR and linear $\left(O\left(n_{\text {state }}\right)\right)$ for SS-SR.

2.2. DMRG-Based (X)MS-CASPT2 Method. In the previous study, we developed the state-specific CASPT2 method using the DMRG-CASSCF wave function as the zeroth-order reference. ${ }^{6,67}$ The central task to implement it was to establish the scheme to obtain the first- to fourth-order reduced density matrices ( $k$-RDMs, $k=1, \ldots, 4)$ of the activespace DMRG wave function. These RDMs are a state-specific (or single-state) object as indicated in the following general form:

$$
D_{j l_{n} \ldots}^{i k m \ldots}(L)=\left\langle\Psi_{L}^{\mathrm{DMRG}}\left|\hat{E}_{j l \ldots}^{i k m \ldots}\right| \Psi_{L}^{\mathrm{DMRG}}\right\rangle
$$

with the spin-summed generator $\hat{E}_{j l n \ldots} i k m \ldots=\sum_{\sigma \tau \rho \ldots}$ $\hat{a}_{i \sigma}^{\dagger} \hat{a}_{k \tau}^{\dagger} \hat{a}_{m \rho}^{\dagger} \ldots \hat{a}_{n \rho} \hat{a}_{l \tau} \hat{a}_{j \sigma}$, where $\sigma, \tau, \rho, \ldots$ refer to the spin indices. The combination of the DMRG and state-specific CASPT2 methods was achieved by using the RDMs of the active-space DMRG wave function in place of those arising in the tensor contraction expressions of the CASPT2 equation and energy.

The 4-RDM is the $O\left(n^{8}\right)$ object, where $n$ means the number of active orbitals. Thus, the computation and memory storage of its full elements become formidable when using a large active space, namely large $n$. In order to mitigate this complexity, we introduced an approximation to the CASPT2 framework, which is realized by replacing the 4-RDM with the approximate one built on-the-fly from low-order (up to third-order) RDMs using the cumulant reconstruction formula. It is referred to as the $\mathrm{cu}(4)$ approximation.

We now turn to the (X)MS extension of DMRG-based CASPT2. The numerical object that is additionally required for this extension is the transition RDMs (TRDMs) of the DMRG references

$$
D_{j l \ldots}^{i k m \ldots}(L, M)=\left\langle\Psi_{L}^{\mathrm{DMRG}}\left|\hat{E}_{j l \ldots}^{i k m \ldots}\right| \Psi_{M}^{\mathrm{DMRG}}\right\rangle \quad(L \neq M)
$$

The RDM (eq 11) and TRDM (eq 12) elements arise as building blocks of the tensor contraction form of the amplitude equation (eq 3) and effective Hamiltonian matrix (eq 4). As similarly done in the state-specific DMRG-CASPT2, the RDM and TRDMs supplied by the DMRG calculations are used for combining DMRG and XMS-CASPT2. The algorithm to compute TRDMs with the DMRG algorithm was recently developed and implemented by three of the authors. ${ }^{85}$

As discussed earlier, the IC basis used in the (X)MSCASPT2 theory is categorized into the MS-MR and SS-SR schemes. Let us here claim that the SS-SR ansatz is the most feasible when based on DMRG references. The reasons are as follows: (1) As indicated earlier, the complexity of the SS-SR scheme scales linearly with $n_{\text {state }}$ and is much lower than that of the MS-MR scheme $\left(O\left(n_{\text {state }}^{3}\right)\right)$. (2) In the absence of the $\mathrm{cu}(4)$ approximation, the highest-order TRDM arising in the SS-SR formalism is 3-TRDM, whereas that in the MS-MR formalism is 4-TRDM, which is required to evaluate the first term of eq 3 . To our best knowledge, the cumulant expansion of TRDMs is not well established. Therefore, choosing the SS-SR scheme that can avoid the cumulant approximation to 4-TRDMs is expedient particularly when the cost of computing the pure 4TRDMs is formidably expensive. It should be, however, noted that this feasibility comes at the price of using inconsistent subsets of the complete IC basis space for the target states and discarding their invariance with respect to the XMS rotation. The state-specific 4-RDM is required for either the SS-SR and MS-MR schemes as the highest-order RDM, and thus the $\mathrm{cu}(4)$ approximation is again exploited for DMRG-based (X)MSCASPT2. In the XMS case, the approximate 4-RDM of the rotated reference $\tilde{L}$ is calculated using the rotated low-order RDMs $\left(\left\langle\tilde{L}\left|\hat{E}_{j}^{i}\right| \tilde{L}\right\rangle,\left\langle\tilde{L}\left|\hat{E}_{j l}^{i k}\right| \tilde{L}\right\rangle\right.$, and $\left.\left\langle\tilde{L}\left|\hat{E}_{j l n}^{i k m}\right| \tilde{L}\right\rangle\right)$ via the cumulant reconstruction formula instead of rotating the reconstructed 4RDM.

2.3. Computer Implementation. The (X)MS-CASPT2 method with the IC basis of the MS-MR and SS-SR schemes was implemented into our in-house program package. The tensor contraction form of the XMS-CASPT2 equations were derived and implemented using the automated code generator developed in refs 59 and 80 . The quantum chemical DMRG program $\mathrm{BLOCK}^{84}$ was used to compute $k$-RDMs and $k$ TRDMs $(k=1,2,3)$ of multiroot DMRG wave functions. ${ }^{85}$ The interface to the full configuration interaction (FCI) program was also implemented to use the pure 4-RDM and 4-TRDM for moderate-size CAS. The resolution-of-identity (or density-fitting) method to accelerate the evaluation of twoelectron integrals ${ }^{94}$ was incorporated into the (X)MS-CASPT2 implementation. The parallel implementation was developed using the standard Message-Passing Interface library and Global Arrays (GA) toolkit ${ }^{95}$ externally linked with the Aggregate Remote Memory Copy Interface compatible library, ARMCIMPI. ${ }^{96}$ The memory allocations for two-electron integrals and amplitudes are distributed across nodes to the data-parallel arrays operated by the GA library. Based on the data distribution, the computation of the amplitude equation (eq 3) is parallelized. The orthonormalization of IC basis with the regulated normalization factors, described in the Appendix, Damped Orthonormalization, is introduced as an approach to alleviate erroneous behaviors caused by the cu(4) approximation.

\section{TEST CALCULATIONS}

Our (X)MS-CASPT2 approach oriented to its combination with multiroot DMRG wave functions was tested by calculating multistate potential energy curves (PECs) of the bond dissociation of the $\mathrm{LiF}$ molecule and the ring-opening and -closing isomerization of the diarylethene derivatives.

3.1. LiF. The original MS-CASPT2 method was developed in the framework of the SS-SR scheme but without the XMS treatment. ${ }^{8}$ The basic performance of the SS-SR based XMSCASPT2 method has not been well examined. The combination of the SS-SR scheme and the XMS-CASPT2 method was also recently shown by Shiozaki et al. on their development of the analytic gradient method. ${ }^{24}$

In order to focus on assessing the performance of the variants of the (X)MS-CASPT2 theory, we examined the covalent-ionic avoided crossing of $\mathrm{LiF}$ as a smallest quasi-degenerate electronic system. The MR character was modeled by a small 

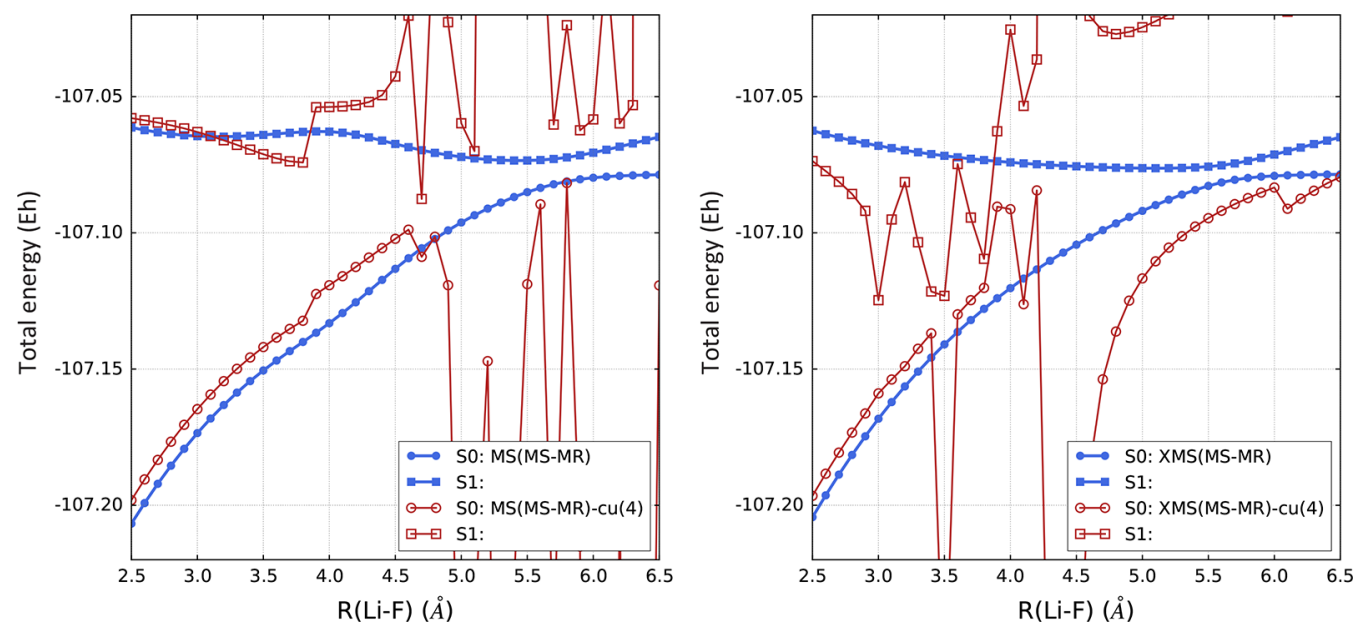

Figure 2. Potential energy curves of the S0 and S1 states of the LiF molecule calculated using the MS-MR framework (eq 1) of the MS-CASPT2 (left panel) and XMS-CASPT2 (right panel) methods with the pure and cu(4)-approximated 4-(T)RDMs. Blue curves: pure 4-(T)RDMs; red curves: cu(4)-approximated 4-(T)RDMs.
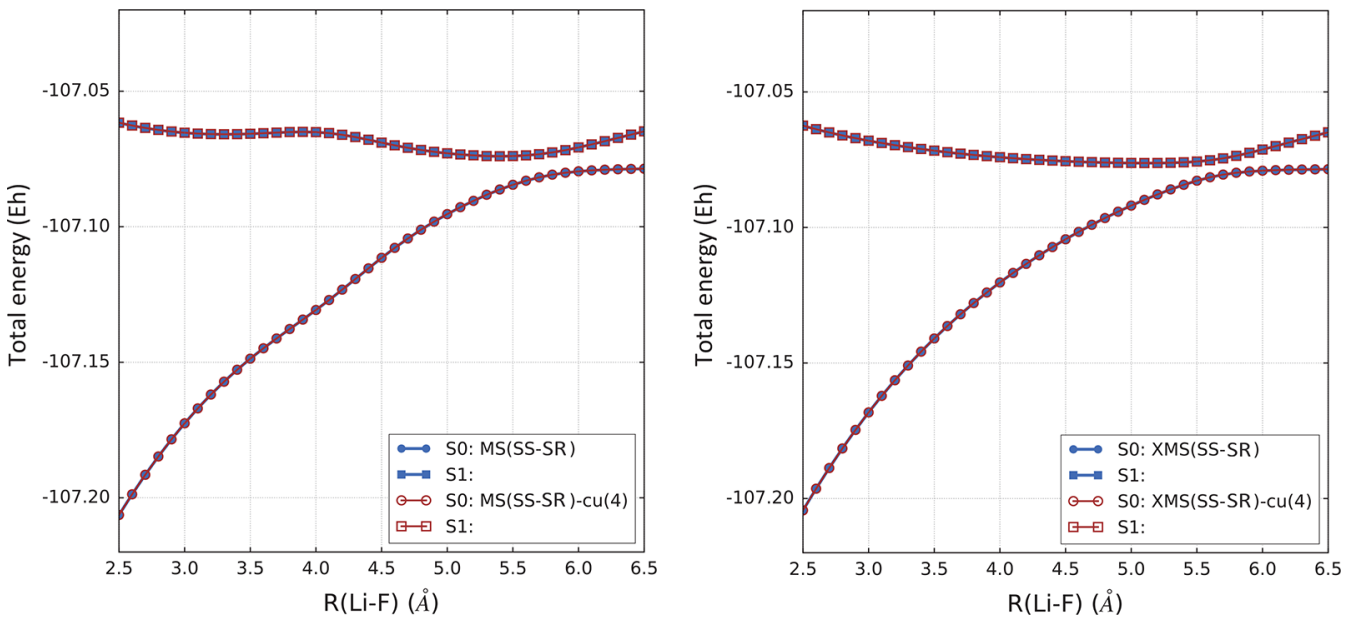

Figure 3. Potential energy curves of the S0 and S1 states of the LiF molecule calculated using the SS-SR framework (eq 2) of the MS-CASPT2 (left panel) and XMS-CASPT2 (right panel) methods with the pure and $\mathrm{cu}(4)$-approximated 4-RDMs. Blue curves: pure 4-RDMs; red curves: cu(4)approximated 4-RDMs.
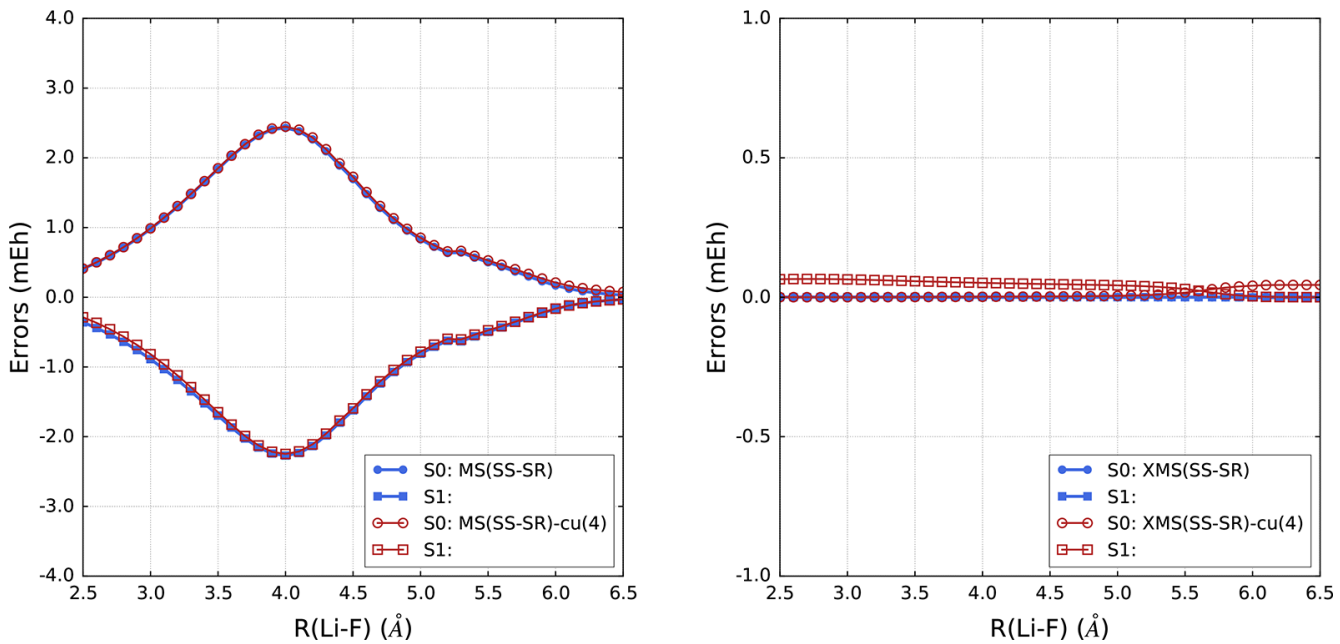

Figure 4. Errors in the potential energy curves of the S0 and S1 states of the LiF molecule calculated using the SS-SR framework (eq 2) of the MSCASPT2 (left panel) and XMS-CASPT2 (right panel) methods with the pure and cu(4)-approximated 4-RDMs, compared to the results obtained by the corresponding MS-MR based methods with the pure 4-(T)RDMs. Blue curves: pure 4-RDMs; red curves: cu(4)-approximated 4-RDMs. 
active space consisting of six active electrons in two $a_{1}$, one $b_{1}$, and one $b_{2}$ active orbitals. We used the conventional CI implementation to obtain the reference wave functions as well as their RDMs and TRDMs including the pure 4-(T)RDMs. The pure and $\mathrm{cu}(4)$-approximated forms of $4-(\mathrm{T}) \mathrm{RDMs}^{97}$ are each used for the (X)MS-CASPT2 calculations. The aug-ccpVTZ basis set $^{98}$ was employed for the atomic orbitals. The core orbitals, $1 \mathrm{~s}$ and $2 \mathrm{~s}$ of the $\mathrm{F}$ atom and $1 \mathrm{~s}$ of the $\mathrm{Li}$ atom, were treated as frozen in the (X)MS-CASPT2 step. The reference states were obtained with the SA-CASSCF procedure considering the two lowest singlet A1 states, designated $\mathrm{S} 0$ and S1, respectively. The MS-CASPT2 and XMS-CASPT2 methods were each carried out in combination with the two types of IC basis, the MS-MR and SS-SR schemes.

Figure 2 shows the PECs obtained by the MS-CASPT2 and XMS-CASPT2 methods with the MS-MR based IC scheme. As noted earlier, the MS-MR scheme describes the (X)MSCASPT2 results with the complete IC basis and requires 4TRDMs. When using the pure 4-(T)RDMs, the PECs resulting from the MS-MR based (X)MS-CASPT2 calculations are regarded as best treated. As previously shown in ref 21, an artificial hump in the $\mathrm{S} 1$ curve yielded by MS-CASPT2 is eliminated with the XMS treatment. Of central interest here is the behavior of the $\mathrm{cu}(4)$ approximation to the MS-MR based (X)MS-CAPT2 theory. As shown in Figure 2, it largely broke down, providing meaningless potential energies. This indicates that the $\mathrm{cu}(4)$ approximation cannot be used in conjunction with the MS-MR scheme.

The PECs obtained by the SS-SR based (X)MS-CASPT2 calculations are displayed in Figure 3. As mentioned before, 4TRDMs are formally unnecessary for these calculations regardless of whether or not using the $\mathrm{cu}(4)$ approximation to 4-RDM. With the use of the pure 4-RDM, the SS-SR based MS-CASPT2 is equivalent to the variant implemented in MOLCAS. Although the SS-SR scheme discards the invariance of IC basis with respect to the XMS rotation, it performed well in combination with the XMS treatment so that the hump of the MS-CASPT2 curve is regularized in the same vein as the MS-MR results. The use of the $\mathrm{cu}(4)$ approximation in this case produced the PECs in good agreement with those obtained using the pure 4-RDM.

In Figure 4, the errors of the SS-SR based (X)MS-CASPT2 total energies are monitored relative to the best treated results that were obtained using the MS-MR scheme with the pure 4(T)RDMs. The errors of the SS-SR based XMS-CASPT2 curves without and with the $\mathrm{cu}(4)$ approximation were within 0.001 and $0.07 m E_{h}$, respectively, indicating that the XMS rotation is well behaved even under the presence of the noninvariance of the SS-SR based IC basis. Interestingly, the nonuse of the XMS treatment substantially enlarged the maximum errors (to $2.4 m E_{h}$ ) and the nonparallelity errors regardless of the $\mathrm{cu}(4)$ approximation.

3.2. Diarylethenes. In the previous assessment, the SS-SR based XMS-CASPT2 has been found to be the most feasible variant when the pure $4-(\mathrm{T}) \mathrm{RDMs}$ are inaccessible. This finding has prompted us to use the SS-SR scheme in our MS extension of DMRG-CASPT2. This combination which is further coupled with the $\mathrm{cu}(4)$ approximation is hereafter referred to as DMRG-cu(4)-XMS-CASPT2. It requires the computation of the pure (T)RDMs of up to third-order in the DMRG process.

Let us here present its application to the multistate calculations of the isomerization of the diarylethene derivatives, which are a widely known type of molecular photochromic systems. ${ }^{99}$ We examined two kinds of the derivatives, (1) the 1,2-bis(2-methyl-5-phenyl-3-thienyl)perfluorocyclopentene ${ }^{100}$ and (2) the 1,2-bis(3-methyl-5-phenyl-2-thienyl)perfluorocyclopentene, ${ }^{101}$ denoted by $\mathrm{N}$-diarylethene and Idiarylethene, respectively, for short (Figure 5). They undergo a reversible photoswitching transformation between two stable isomers: open-ring and closed-ring.

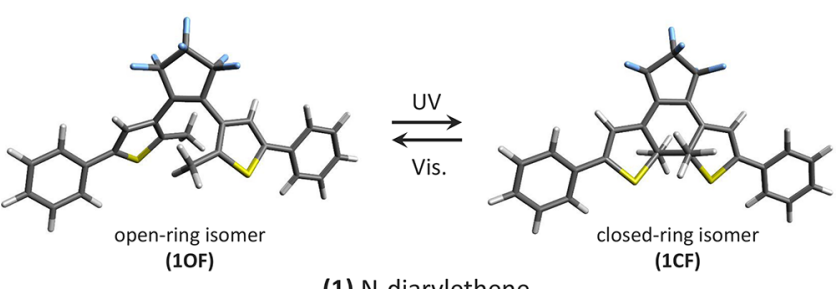

(1) N-diarylethene

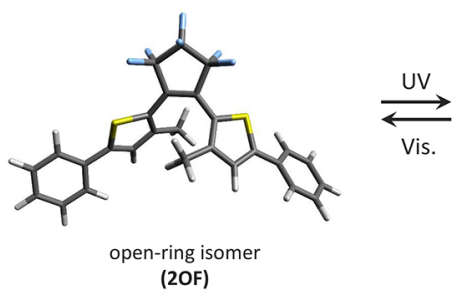

(2) I-diarylethene

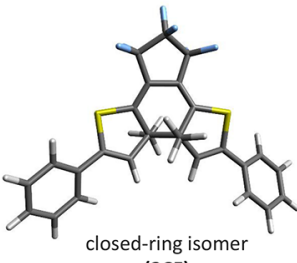

(2CF)
Figure 5. B3LYP-D3/def2-TZVPP-optimized structures of open-ring $(\mathrm{OF})$ and closed-ring (CF) isomers of (1) N-diarylethene and (2) Idiarylethene considered in this study. They undergo a ring-opening and -closing reaction upon $\mathrm{UV}$ and visible irradiation.

The molecular structures of the open- and closed-ring isomers were determined by the geometry optimizations at the B3LYP-D3/def2-TZVPP level of theory ${ }^{102-104}$ for the singlet ground state. The intermediate structures of the isomerization were modeled by interpolating the two optimized isomeric geometries. The progress of the isomerization is denoted by the reaction coordinate $F$, ranging from 1 to 10 , where the coordinates 1 and 10 correspond to the optimized open- and close-ring isomers, respectively, denoted as $i \mathrm{OF}$ and $i \mathrm{CF}(i=1$ for $\mathrm{N}$-diarylethene and $i=2$ for I-diarylethene), respectively. All of the geometries are compiled in the data files of the Supporting Information.

The DMRG-CASSCF/XMS-CASPT2 calculations were performed using the def2-SVP basis set. ${ }^{105}$ The reference wave functions were calculated using the DMRG-SA-CASSCF method with the CAS $(26 \mathrm{e}, 240)$, which accounts for the full $\pi$ valence active space. The active orbitals, which take a localized form as used for the DMRG lattice sites, are shown in Figure 6. The number of the spin-adapted renormalized basis in the DMRG calculations was 256 . We considered the four lowestlying singlet states in the multistate PEC calculations. As mentioned before, the pure 1-, 2-, and 3-(T)RDMs were calculated from the DMRG-SA-CASSCF wave functions, and the 4-RDMs arising in the SS-SR scheme were treated with the $\mathrm{cu}(4)$ approximation. Note again that the theory is free from 4TRDMs. The SS-SR based XMS-CASPT2 calculations were carried out with an imaginary level shift ${ }^{106}$ and an IPEA shift ${ }^{107}$ set to $i 0.1$ and 0.10 au, respectively. In addition, the partial diagonal approximation (Appendix, Damped Orthonormalization) to $c c v v$-type amplitudes was used together with the 

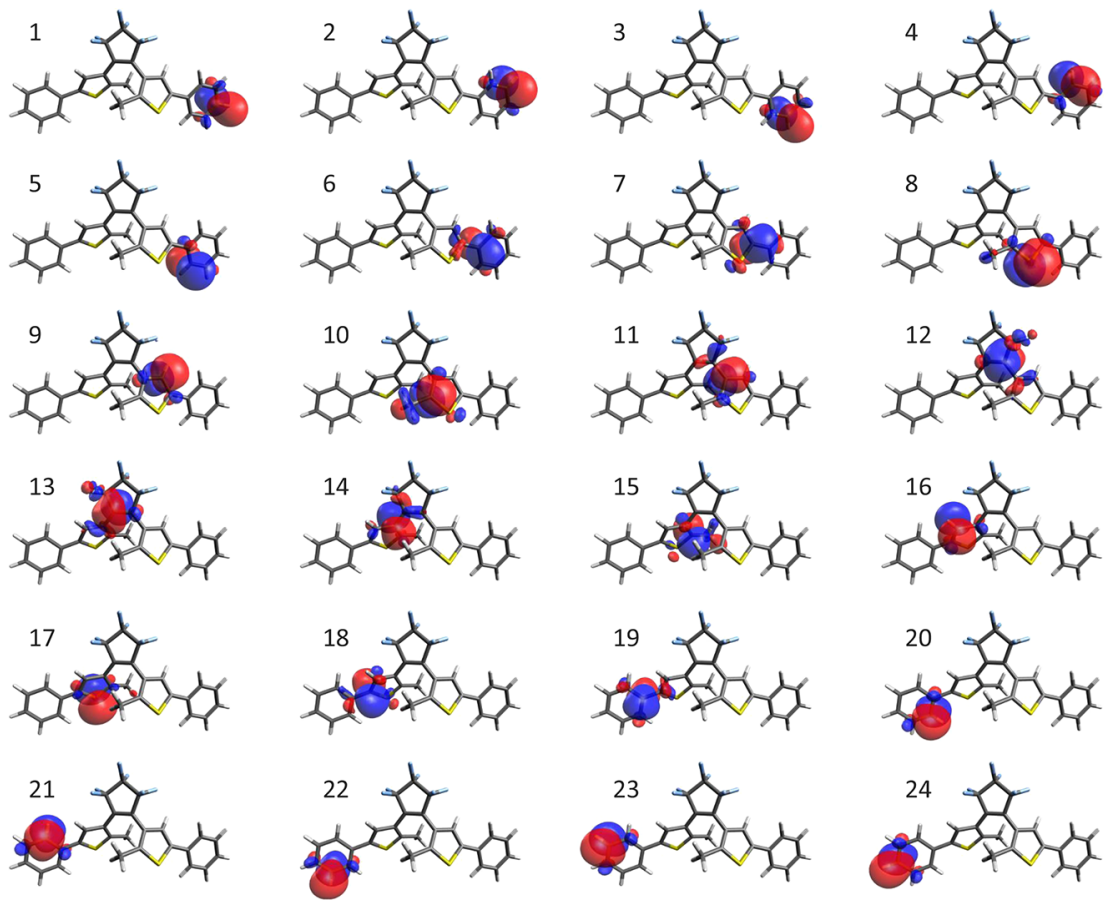

Figure 6. Localized DMRG-CASSCF active orbitals of the open-ring N-diarylethene in the ordering on the DMRG lattice sites.
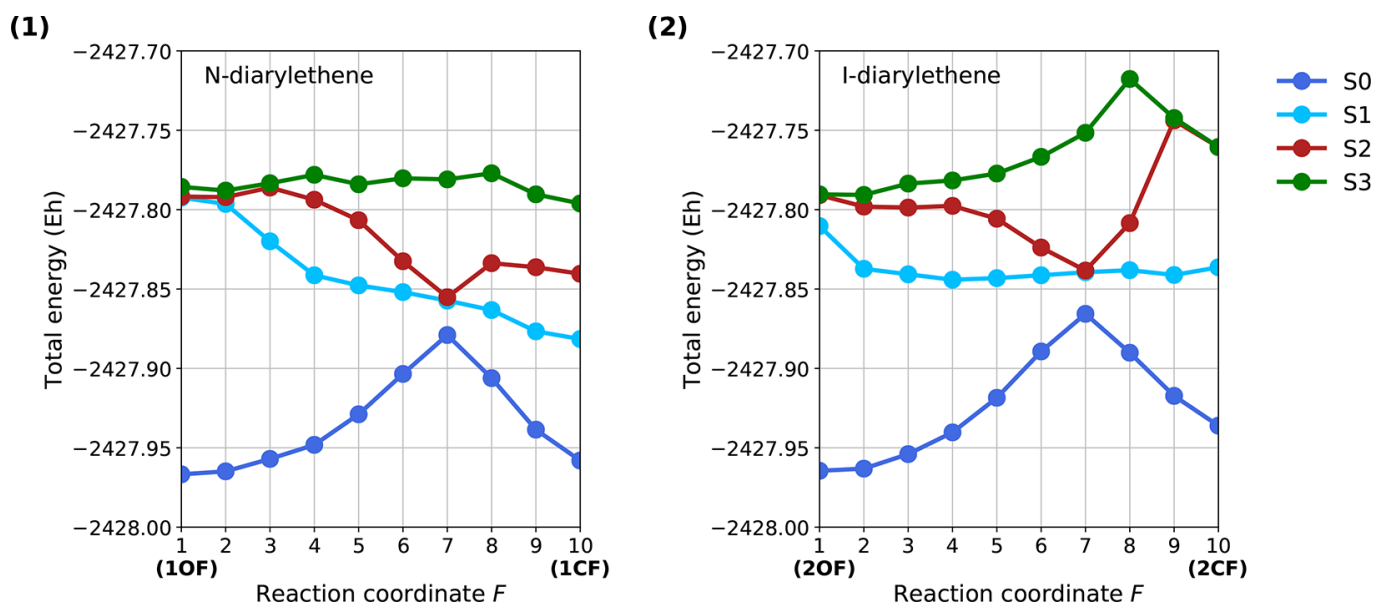

Figure 7. Total energies of the S0-S3 states of (1) N-diarylethene and (2) I-diarylethene determined by the four-state DMRG-cu(4)-XMS-CASPT2 calculations with CAS $(26 \mathrm{e}, 24 \mathrm{o})$ accouting for the full $\pi$ active space. The plots are given as a function of the reaction coordinate $F$. $F=1$ and $F=10$ correspond to the DFT-optimized open-ring and closed-ring isomers, respectively. The def2-SVP basis set was used.

damped orthonormalization (Appendix, Partial Diagonal Approximation) with the shift parameter $\epsilon=0.01$ au.

The multistate PECs of N-diarylethene and I-diarylethene obtained by the DMRG-cu(4)-XMS-CASPT2 calculations are shown in Figure 7. The vertical transition energies from the ground state to the S1 and S2 states are compiled in Table 2 along with the oscillator strengths. For comparison, the experimental values of the absorption energies of the open and closed isomers in the hexane solution are included in the table. ${ }^{100,101}$ According to the calculated oscillator strengths, the S1 and S2 states for both the diarylethene derivatives seemingly correspond to what are known as the optically allowed $1 \mathrm{~B}$ and forbidden $2 \mathrm{~A}$ states, respectively. ${ }^{99}$ The calculated transition energies of the $1 \mathrm{~B}$ state for the geometries close to $1 \mathrm{OF}, 1 \mathrm{CF}$, $2 \mathrm{OF}$, and $2 \mathrm{CF}$ are all in reasonably good agreement with the experimental absorption energies. By checking the off-diagonal elements of the effective Hamiltonian $H_{M L}^{\text {eff }}$ (eq 4), we confirmed that the avoided crossing at the reaction coordinate 7 is characterized by the mixing of the So (1A) and S2 (2A) states. This indicates that the characters of the S0 and S2 states intercross during the isomerization process.

Experimentally, the ring-opening reaction of the perfluorocyclopentene diarylethene derivatives is activated under the steady state irradiation with visible light but with an extremely low reaction yield. However, the recent laser photolysis carried out by Miyasaka et al. showed that the two-photon absorption laser treatment significantly increases its quantum yield by a factor of ca. $400 .^{108}$ This can be explained using the calculated PECs (Figure 7) as follows. The one-photon absorption upon the steady state irradiation excites the closed isomer to the allowed S1 state. The S1 curve is not sufficiently downhill to promote the ring-opening reaction, so that it has a short lifetime. The two-photon absorption process somewhat leads to the production of the high-energy S2 state. The presence of an 
Table 2. Relative Energies (in nm) of the S1 and S2 States, Denoted 1B and 2A, Respectively, with Respect to the Total Energies of the So State, Calculated at the DMRG-cu(4)-XMS-CASPT2(26e,24o)/def2-SVP Level of Theory, Along with the Experimental Values of the Absorption Energies in the Hexane Solution ${ }^{a}$

\begin{tabular}{|c|c|c|c|c|c|c|c|c|c|c|}
\hline \multirow{4}{*}{$\begin{array}{l}\text { coordinate } \\
1 \text { (open) }\end{array}$} & \multicolumn{5}{|c|}{ N-diarylethene } & \multicolumn{5}{|c|}{ I-diarylethene } \\
\hline & \multicolumn{3}{|c|}{ S1 (1B) } & \multirow{2}{*}{\multicolumn{2}{|c|}{$\frac{\text { S2 }(2 \mathrm{~A})}{\text { calc. }}$}} & \multicolumn{3}{|c|}{ S1 (1B) } & \multirow{2}{*}{\multicolumn{2}{|c|}{$\frac{\text { S2 }(2 \mathrm{~A})}{\text { calc. }}$}} \\
\hline & \multicolumn{2}{|c|}{ calc. } & \multirow{2}{*}{$\begin{array}{r}\text { expt. }^{b} \\
280\end{array}$} & & & \multicolumn{2}{|c|}{ calc. } & \multirow{2}{*}{$\begin{array}{r}\operatorname{expt.}^{c} \\
370\end{array}$} & & \\
\hline & 261 & $(0.0001)$ & & 260 & $(0.0003)$ & 296 & $(0.2803)$ & & 262 & $(0.0009)$ \\
\hline 2 & 270 & $(0.0015)$ & & 264 & $(0.0001)$ & 361 & $(0.5175)$ & & 276 & $(0.0158)$ \\
\hline 3 & 332 & $(0.0133)$ & & 267 & $(0.0001)$ & 402 & $(0.4006)$ & & 293 & $(0.0102)$ \\
\hline 4 & 427 & $(0.0211)$ & & 295 & $(0.0010)$ & 474 & $(0.2588)$ & & 319 & $(0.0049)$ \\
\hline 5 & 561 & $(0.0102)$ & & 373 & $(0.0016)$ & 605 & $(0.1207)$ & & 404 & $(0.0017)$ \\
\hline 6 & 884 & $(0.0067)$ & & 642 & $(0.0018)$ & 949 & $(0.0278)$ & & 696 & $(0.0005)$ \\
\hline 7 & 2104 & $(0.0004)$ & & 1914 & $(0.0003)$ & 1740 & $(0.0013)$ & & 1669 & $(0.0011)$ \\
\hline 8 & 1063 & $(0.0750)$ & & 629 & $(0.0012)$ & 876 & $(0.0113)$ & & 558 & $(0.0010)$ \\
\hline 9 & 736 & $(0.2064)$ & & 445 & $(0.0003)$ & 598 & $(0.0726)$ & & 263 & $(0.0004)$ \\
\hline 10 (closed) & 595 & $(0.3068)$ & 575 & 387 & $(0.0000)$ & 457 & $(0.1743)$ & 438 & 260 & $(0.0004)$ \\
\hline
\end{tabular}

efficient state-crossing route that connects it to the stable ringopen isomer state $(2 \mathrm{~A} \rightarrow 1 \mathrm{~A})$ seem to be highly favorable for the promotion of the reaction, which should contribute to an increase in the quantum yield. In this light, the present DMRG$\mathrm{cu}(4)-X M S-C A S P T 2$ results are consistent with the experimental observations.

\section{CONCLUSIONS}

We have developed the MS extension of the DMRG-CASPT2 method of refs 66 and 67 as an efficient MR theory for studying excited states with a large-size active space. It is based on the MS-CASPT2 theory introduced by Finley et al. and the XMSCASPT2 theory developed by Shiozaki et al. as an incorporation of Granovsky's XMS method into the MSCASPT2 theory. The SS-SR based IC basis scheme has been found the most feasible variant of the (X)MS-CASPT2 theory when using the DMRG reference wave functions. The feasibility of the SS-SR scheme is 2-fold: (1) we can avoid the direct use of the $\mathrm{cu}(4)$ approximation to 4-TRDM and (2) its computational complexity grows at a linear rate with increasing number of the reference states instead of a cubic rate that should be caused in the MS-MR scheme. The $\mathrm{cu}(4)$ approximation to 4-RDM has been exploited as similarly done in the state-specific DMRG-cu(4)-CASPT2 approach. In the DMRG process, the pure (T)RDMs of up to third-order are computed. We demonstrated the performance of the DMRG$\mathrm{cu}(4)$-XMS-CASPT2 implementation by calculating the multistate PECs of the photochemical systems using a large active space, $(26 \mathrm{e}, 240)$. It was shown that the XMS rotation is practically well behaved despite the noninvariance of the SS-SR based IC basis with it. Shiozaki et al. recently reported an elaborate technique to implement the analytic gradient method of the (X)MS-CASPT2 theory. ${ }^{23-27}$ The incorporation of this technology into the DMRG-XMS-CASPT2 method now comes into our sight.

\section{APPENDICES}

\section{Damped Orthonormalization}

The $\mathrm{cu}(4)$ approximation essentially introduces errors in the energy denominators of the CASPT2 theory. The errors may in some case give rise to too small denominators (so-called "false intruders") and appreciable discontinuity in potential curves. ${ }^{79}$ This "false intruders" issue can be suppressed to some extent by incorporating the conventional intruder state avoidance techniques, such as the level-shift, ${ }^{109}$ and imaginary level-shift correction $^{106}$ methods, as well as IPEA shift treatment. ${ }^{107}$ Pierloot et al. very recently presented the detailed assessment of the cu(4)-CASPT2 method. ${ }^{56}$ Here we present a complementary approach to regulate the intruders arising from $\mathrm{cu}(4)$ approximation.

Let the expansion of the first-order wave function of the CASPT2 theory be written as $\left|\Psi^{(1)}\right\rangle=\sum_{\Omega} T_{\Omega}|\Omega\rangle$ where the IC basis is denoted $|\Omega\rangle$. The IC basis is orthonormalized by the linear transformation as

$$
|i\rangle=\sum_{\Omega}|\Omega\rangle U_{\Omega i} f_{i}
$$

with the normalization factor $f_{i}$ given by $1 / \sqrt{s_{i}}$, where $s_{i}$ and $U_{\Omega i}$ are the eigenvalues and eigenvectors of $S_{\Omega \Pi}$, respectively; $\mathbf{S}$ is the overlap matrix in IC basis given by $S_{\Omega \Pi}=\langle\Omega \mid \Pi\rangle$. In this procedure, the orthonormalized IC basis $|i\rangle$ with negligible $s_{i}$ less than a threshold (e.g., $10^{-8}$ a.u.) are truncated for the sake of numerical stability.

Our numerical tests showed that the cumulant error is excessively amplified in the denominator element associated with $|i\rangle$ with fairly small $s_{i}$ (near above the truncation threshold). To mitigate this error amplification, we introduce the regulation of $1 / \sqrt{s_{i}}$ with the following imaginary level-shift transformation:

$$
f_{i} \simeq \operatorname{Re}\left[\frac{1}{\sqrt{s_{i}}+i \epsilon}\right]=\frac{\sqrt{s_{i}}}{s_{i}+\epsilon^{2}}
$$

where $\epsilon$ is the user-specified parameter and $f_{i}$ converges to $1 / \sqrt{s_{i}}$ for $\epsilon \rightarrow 0$. This regulation, referred to as damped orthonormalization, is carried out for constructing the IC basis of the semi-internal cooo and ooov excitation types, where $c, o$, and $v$ mean core, active, and external orbital, and cooo (or ooov) denotes the class of $(c, o) \rightarrow(o, o)$ (or $(o, o) \rightarrow(o, v))$ excitation.

\section{Partial Diagonal Approximation}

The number of the amplitude coefficients, $T_{\Omega}$, of eqs 1 and 2 (discarding the state indices $L$ and $N)$, is $O\left[\left(n_{c}+n_{o}\right)^{2}\left(n_{c}+\right.\right.$ $\left.\left.n_{v}\right)^{2}\right]$, where $n_{\mathcal{c}}, n_{o}$, and $n_{v}$ are the number of core, active, and external orbitals. In practice, $n_{c}$ and $n_{v}$ are in most cases much larger than $n_{o}$; thus, the $c c v v$-type elements $\left(O\left[n_{c}{ }^{2} n_{v}{ }^{2}\right]\right)$ use the largest part of memory for the storage of the amplitude. The 
steeply-growing memory requirement for them hinders CASPT2 applications for large molecular systems.

In our implementation, we introduced an option to apply the diagonal approximation of CASPT2-D ${ }^{6}$ specifically to the $c c v v$ type amplitude so as to eliminate its storage. With this option, the coefficients of $c c v v$ type are noniteratively determined to be as follows:

$$
T_{x y a b}=\frac{2(x a \mid y b)-(y a \mid x b)}{\epsilon_{x}+\epsilon_{y}-\epsilon_{a}-\epsilon_{b}}
$$

where we assume that the core and external orbitals are canonical orbitals, satisfying $\langle x \mid \hat{f} y\rangle=0(x \neq y)$ and $\langle a \mid \hat{f} b\rangle=0$ $(a \neq b)$. Equation 15 takes the same form as the first-order Møller-Plesset amplitude; ( $p q \mid r s)$ is the two-electron repulsion integral in Mulliken notation, and $e_{p}$ is the digaonal element of the Fock matrix, i.e., $\langle p|\hat{f}| p\rangle$. The $c c v v$-type amplitude is fixed by eq 15 and is computed on-the-fly where they are necessary for evaulating the energy and amplitude equation. The coefficients of the other amplitude types are stored in memory and optimized by solving the amplitude equation, in which $T_{x y a b}$ with the diagonal approximation is involved as fixed parameters. Errors caused by this approximation are usually small in relative energies, such as excitation energies.

\section{ASSOCIATED CONTENT}

\section{S Supporting Information}

The Supporting Information is available free of charge on the ACS Publications website at DOI: 10.1021/acs.jctc.7b00735.

Total energies for LiF; DMRG-SA-CASSCF and DMRG-cu(4)-XMS-CASPT2 energies for $\mathrm{N}$-diarylethene and I-diarylethene. XLS)

Geometric data of the modeled reaction of $N$-diarylethene. (XYZ)

Geometric data of the modeled reaction of I-diarylethene. (XYZ)

\section{AUTHOR INFORMATION}

\section{Corresponding Author}

*E-mail: yanait@ims.ac.jp.

\section{ORCID}

Yuki Kurashige: 0000-0003-4652-8153

Sandeep Sharma: 0000-0002-6598-8887

\section{Present Address}

$\triangle$ Max Planck Institute for Chemical Energy Conversion, Stiftstr. 32-34, D-45470, Mülheim an der Ruhr, Germany.

\section{Funding}

This work was supported partially by JSPS KAKENHI Grant Nos. JP15H01097, JP16H04101, and JP17H05274 for T.Y. and National Natural Science Foundation of China (21501189) and Grant-in-Aid for Invitational Fellowship for Research in Japan (L16525) for X.-G.X. J.Ch. was supported by the Grant Agency of the Czech Republic (Grant No. 15-19143S) and institutional support RVO 61388963 (CAS).

\section{Notes}

The authors declare no competing financial interest.

\section{ACKNOWLEDGMENTS}

The authors thank Professor Garnet Kin-Lic Chan for valuable comments.

\section{REFERENCES}

(1) Roos, B. O. Theoretical Studies of Electronically Excited States of Molecular Systems Using Multiconfigurational Perturbation Theory. Acc. Chem. Res. 1999, 32, 137-144.

(2) Hirao, K. Recent advances in multireference methods; World Scientific: Singapore, 1999.

(3) Buenker, R. J.; Peyerimhoff, S. D. Individualized configuration selection in CI calculations with subsequent energy extrapolation. Theor. Chim. Acta. 1974, 35, 33-58.

(4) Werner, H.-J.; Knowles, P. J. An efficient internally contracted multiconfiguration-reference configuration interaction method. J. Chem. Phys. 1988, 89, 5803-5814.

(5) Knowles, P. J.; Werner, H.-J. Internally contracted multiconfiguration-reference configuration interaction calculations for excited states. Theor. Chim. Acta 1992, 84, 95-103.

(6) Andersson, K.; Malmqvist, P.-Å.; Roos, B. O.; Sadlej, A. J.; Wolinski, K. Second-order perturbation theory with a CASSCF reference function. J. Phys. Chem. 1990, 94, 5483-5488.

(7) Andersson, K.; Malmqvist, P.-Å.; Roos, B. O. Second-order perturbation theory with a complete active space self-consistent field reference function. J. Chem. Phys. 1992, 96, 1218-1226.

(8) Finley, J.; Malmqvist, P.-Å.; Roos, B. O.; Serrano-Andrés, L. The multi-state CASPT2 method. Chem. Phys. Lett. 1998, 288, 299-306.

(9) Malmqvist, P.-Å.; Pierloot, K.; Shahi, A. R. M.; Cramer, C. J.; Gagliardi, L. The restricted active space followed by second-order perturbation theory method: theory and application to the study of $\mathrm{CuO} 2$ and $\mathrm{Cu} 2 \mathrm{O} 2$ systems. J. Chem. Phys. 2008, 128, 204109.

(10) Hirao, K. Multireference Møller-Plesset method. Chem. Phys. Lett. 1992, 190, 374-380.

(11) Nakano, H. Quasidegenerate perturbation theory with multiconfigurational self-consistent-field reference functions. J. Chem. Phys. 1993, 99, 7983-7992.

(12) Nakano, H. MCSCF reference quasidegenerate perturbation theory with Epstein-Nesbet partitioning. Chem. Phys. Lett. 1993, 207, 372-378.

(13) Angeli, C.; Cimiraglia, R.; Evangelisti, S.; Leininger, T.; Malrieu, J.-P. Introduction of n-electron valence states for multireference perturbation theory. J. Chem. Phys. 2001, 114, 10252-10264.

(14) Angeli, C.; Borini, S.; Cestari, M.; Cimiraglia, R. A quasidegenerate formulation of the second order $\mathrm{n}$-electron valence state perturbation theory approach. J. Chem. Phys. 2004, 121, 40434049.

(15) Fink, R. F. Two new unitary-invariant and size-consistent perturbation theoretical approaches to the electron correlation energy. Chem. Phys. Lett. 2006, 428, 461-466.

(16) Fink, R. F. The multi-reference retaining the excitation degree perturbation theory: A size-consistent, unitary invariant, and rapidly convergent wavefunction based ab initio approach. Chem. Phys. 2009, $356,39-46$.

(17) Sharma, S.; Knizia, G.; Guo, S.; Alavi, A. Combining Internally Contracted States and Matrix Product States To Perform Multireference Perturbation Theory. J. Chem. Theory Comput. 2017, 13, 488-498.

(18) Sharma, S.; Alavi, A. Multireference linearized coupled cluster theory for strongly correlated systems using matrix product states. $J$. Chem. Phys. 2015, 143, 102815.

(19) Celani, P.; Werner, H.-J. Multireference perturbation theory for large restricted and selected active space reference wave functions. J. Chem. Phys. 2000, 112, 5546-5557.

(20) Celani, P.; Werner, H.-J. Analytical energy gradients for internally contracted second-order multireference perturbation theory. J. Chem. Phys. 2003, 119, 5044-5057.

(21) Shiozaki, T.; Györffy, W.; Celani, P.; Werner, H.-J. Communication: Extended multi-state complete active space secondorder perturbation theory: Energy and nuclear gradients. J. Chem. Phys. 2011, 135, 081106.

(22) Györffy, W.; Shiozaki, T.; Knizia, G.; Werner, H.-J. Analytical energy gradients for second-order multireference perturbation theory using density fitting. J. Chem. Phys. 2013, 138, 104104. 
(23) MacLeod, M. K.; Shiozaki, T. Communication: Automatic code generation enables nuclear gradient computations for fully internally contracted multireference theory. J. Chem. Phys. 2015, 142, 051103.

(24) Vlaisavljevich, B.; Shiozaki, T. Nuclear Energy Gradients for Internally Contracted Complete Active Space Second-Order Perturbation Theory: Multistate Extensions. J. Chem. Theory Comput. 2016, 12, 3781-3787.

(25) Shiozaki, T.; Yanai, T. Hyperfine Coupling Constants from Internally Contracted Multireference Perturbation Theory. J. Chem. Theory Comput. 2016, 12, 4347-4351.

(26) Park, J. W.; Shiozaki, T. Analytical Derivative Coupling for Multistate CASPT2 Theory. J. Chem. Theory Comput. 2017, 13, 25612570.

(27) Park, J. W.; Shiozaki, T. J. Chem. Thoery Comput. 2017, 13, 3676.

(28) Ruedenberg, K.; Schmidt, M.; Gilbert, M.; Elbert, S. Are atoms intrinsic to molecular electronic wavefunctions? I. The FORS model. Chem. Phys. 1982, 71, 41-49.

(29) Roos, B. O. The complete active space self-consistent field method and its applications in electronic structure calculations. Adv. Chem. Phys. 1987, 69, 399-445.

(30) Bloch, C. Sur la théorie des perturbations des états liés. Nucl. Phys. 1958, 6, 329-347.

(31) Primas, H. Generalized Perturbation Theory in Operator Form. Rev. Mod. Phys. 1963, 35, 710-711.

(32) Brandow, B. H. Linked-Cluster Expansions for the Nuclear Many-Body Problem. Rev. Mod. Phys. 1967, 39, 771-828.

(33) Kirtman, B. Variational Form of Van Vleck Degenerate Perturbation Theory with Particular Application to Electronic Structure Problems. J. Chem. Phys. 1968, 49, 3890-3894.

(34) Certain, P. R.; Hirschfelder, J. O. New Partitioning Perturbation Theory. I. General Formalism. J. Chem. Phys. 1970, 52, 5977-5987.

(35) Kirtman, B. Simultaneous calculation of several interacting electronic states by generalized Van Vleck perturbation theory. J. Chem. Phys. 1981, 75, 798-808.

(36) Shavitt, I.; Redmon, L. T. Quasidegenerate perturbation theories. A canonical van Vleck formalism and its relationship to other approaches. J. Chem. Phys. 1980, 73, 5711-5717.

(37) Zaitsevskii, A.; Malrieu, J.-P. Multi-partitioning quasidegenerate perturbation theory. A new approach to multireference Møller-Plesset perturbation theory. Chem. Phys. Lett. 1995, 233, 597-604.

(38) Malrieu, J.-P.; Heully, J.-L.; Zaitsevskii, A. Multiconfigurational second-order perturbative methods: Overview and comparison of basic properties. Theor. Chim. Acta 1995, 90, 167-187.

(39) Granovsky, A. A. Extended multi-configuration quasi-degenerate perturbation theory: The new approach to multi-state multi-reference perturbation theory. J. Chem. Phys. 2011, 134, 214113.

(40) White, S. R. Density matrix formulation for quantum renormalization groups. Phys. Rev. Lett. 1992, 69, 2863-2866.

(41) White, S. R. Density-matrix algorithms for quantum renormalization groups. Phys. Rev. B: Condens. Matter Mater. Phys. 1993, 48, 10345-10356.

(42) White, S. R; Martin, R. L. Ab initio quantum chemistry using the density matrix renormalization group. J. Chem. Phys. 1999, 110, 4127-4130.

(43) Chan, G. K.-L.; Sharma, S. The density matrix renormalization group in quantum chemistry. Annu. Rev. Phys. Chem. 2011, 62, 465481.

(44) Sharma, S.; Sivalingam, K.; Neese, F.; Chan, G. K.-L. Lowenergy spectrum of iron-sulfur clusters directly from many-particle quantum mechanics. Nat. Chem. 2014, 6, 927-933.

(45) Sharma, S.; Yanai, T.; Booth, G. H.; Umrigar, C. J.; Chan, G. K.L. Spectroscopic accuracy directly from quantum chemistry: Application to ground and excited states of beryllium dimer. J. Chem. Phys. 2014, 140, 104112.

(46) Wouters, S.; Jiménez-Hoyos, C. A.; Sun, Q.; Chan, G. K.-L. A Practical Guide to Density Matrix Embedding Theory in Quantum Chemistry. J. Chem. Theory Comput. 2016, 12, 2706-2719.
(47) Li, Z.; Chan, G. K.-L. Spin-Projected Matrix Product States: Versatile Tool for Strongly Correlated Systems. J. Chem. Theory Comput. 2017, 13, 2681-2695.

(48) Marti, K. H.; Ondík, I. M.; Moritz, G.; Reiher, M. Density matrix renormalization group calculations on relative energies of transition metal complexes and clusters. J. Chem. Phys. 2008, 128, 014104.

(49) Knecht, S.; Legeza, Ö.; Reiher, M. Communication: Fourcomponent density matrix renormalization group. J. Chem. Phys. 2014, 140, 041101.

(50) Keller, S.; Dolfi, M.; Troyer, M.; Reiher, M. An efficient matrix product operator representation of the quantum chemical Hamiltonian. J. Chem. Phys. 2015, 143, 244118.

(51) Freitag, L.; Knecht, S.; Angeli, C.; Reiher, M. Multireference Perturbation Theory with Cholesky Decomposition for the Density Matrix Renormalization Group. J. Chem. Theory Comput. 2017, 13, 451-459.

(52) Zgid, D.; Nooijen, M. On the spin and symmetry adaptation of the density matrix renormalization group method. J. Chem. Phys. 2008, $128,014107$.

(53) Szalay, S.; Pfeffer, M.; Murg, V.; Barcza, G.; Verstraete, F.; Schneider, R.; Legeza, Ö. Tensor product methods and entanglement optimization for $\mathrm{ab}$ initio quantum chemistry. Int. J. Quantum Chem. 2015, 115, 1342-1391.

(54) Wouters, S.; Poelmans, W.; De Baerdemacker, S.; Ayers, P. W.; Van Neck, D. CheMPS2: Improved DMRG-SCF routine and correlation functions. Comput. Phys. Commun. 2015, 191, 235-237.

(55) Wouters, S.; Van Speybroeck, V.; Van Neck, D. DMRGCASPT2 study of the longitudinal static second hyperpolarizability of all-trans polyenes. J. Chem. Phys. 2016, 145, 054120.

(56) Phung, Q. M.; Wouters, S.; Pierloot, K. Cumulant Approximated Second-Order Perturbation Theory Based on the Density Matrix Renormalization Group for Transition Metal Complexes: A Benchmark Study. J. Chem. Theory Comput. 2016, 12, $4352-4361$.

(57) Kurashige, Y.; Chan, G. K.-L.; Yanai, T. Entangled quantum electronic wavefunctions of the $\mathrm{Mn} 4 \mathrm{CaO} 5$ cluster in photosystem II. Nat. Chem. 2013, 5, 660-666.

(58) Chalupský, J.; Rokob, T. A.; Kurashige, Y.; Yanai, T.; Solomon, E. I.; Rulišsek, L.; Srnec, M. Reactivity of the Binuclear Non-Heme Iron Active Site of 9 Desaturase Studied by Large-Scale Multireference Ab Initio Calculations. J. Am. Chem. Soc. 2014, 136, 15977-15991.

(59) Saitow, M.; Kurashige, Y.; Yanai, T. Fully Internally Contracted Multireference Configuration Interaction Theory Using Density Matrix Renormalization Group: A Reduced-Scaling Implementation Derived by Computer-Aided Tensor Factorization. J. Chem. Theory Comput. 2015, 11, 5120-5131.

(60) Yanai, T.; Kurashige, Y.; Mizukami, W.; Chalupský, J.; Lan, T. N.; Saitow, M. Density matrix renormalization group for ab initio Calculations and associated dynamic correlation methods: A review of theory and applications. Int. J. Quantum Chem. 2015, 115, 283-299.

(61) Veis, L.; Antalík, A.; Brabec, J.; Neese, F.; Legeza, Ã.; Pittner, J. Coupled Cluster Method with Single and Double Excitations Tailored by Matrix Product State Wave Functions. J. Phys. Chem. Lett. 2016, 7, 4072-4078.

(62) Östlund, S.; Rommer, S. Thermodynamic Limit of Density Matrix Renormalization. Phys. Rev. Lett. 1995, 75, 3537-3540.

(63) Verstraete, F.; Porras, D.; Cirac, J. I. Density Matrix Renormalization Group and Periodic Boundary Conditions: A Quantum Information Perspective. Phys. Rev. Lett. 2004, 93, 227205.

(64) Schollwöck, U. The density-matrix renormalization group in the age of matrix product states. Ann. Phys. 2011, 326, 96-192. January 2011 Special Issue.

(65) Chan, G. K.-L.; Keselman, A.; Nakatani, N.; Li, Z.; White, S. R Matrix product operators, matrix product states, and ab initio density matrix renormalization group algorithms. J. Chem. Phys. 2016, 145, 014102.

(66) Kurashige, Y.; Yanai, T. Second-order perturbation theory with a density matrix renormalization group self-consistent field reference 
function: Theory and application to the study of chromium dimer. J. Chem. Phys. 2011, 135, 094104.

(67) Kurashige, Y.; Chalupský, J.; Lan, T. N.; Yanai, T. Complete active space second-order perturbation theory with cumulant approximation for extended active-space wavefunction from density matrix renormalization group. J. Chem. Phys. 2014, 141, 174111.

(68) Ghosh, D.; Hachmann, J.; Yanai, T.; Chan, G. K.-L. Orbital optimization in the density matrix renormalization group, with applications to polyenes and $\beta$-carotene. J. Chem. Phys. 2008, 128, 144117.

(69) Zgid, D.; Nooijen, M. The density matrix renormalization group self-consistent field method: Orbital optimization with the density matrix renormalization group method in the active space. J. Chem. Phys. 2008, 128, 144116.

(70) Sun, Q.; Yang, J.; Chan, G. K.-L. A general second order complete active space self-consistent-field solver for large-scale systems. Chem. Phys. Lett. 2017, 683, 291.

(71) Ma, Y.; Ma, H. Assessment of various natural orbitals as the basis of large active space density-matrix renormalization group calculations. J. Chem. Phys. 2013, 138, 224105.

(72) Ma, Y.; Knecht, S.; Keller, S.; Reiher, M. Second-Order SelfConsistent-Field Density-Matrix Renormalization Group. J. Chem. Theory Comput. 2017, 13, 2533-2549.

(73) Kurashige, Y. Multireference electron correlation methods with density matrix renormalisation group reference functions. Mol. Phys. 2014, 112, 1485-1494.

(74) Liu, F.; Kurashige, Y.; Yanai, T.; Morokuma, K. Multireference Ab Initio Density Matrix Renormalization Group (DMRG)-CASSCF and DMRG-CASPT2 Study on the Photochromic Ring Opening of Spiropyran. J. Chem. Theory Comput. 2013, 9, 4462-4469.

(75) Kurashige, Y.; Yanai, T. Theoretical Study of the $\pi \rightarrow \pi^{*}$ Excited States of Oligoacenes: A Full $\pi$-Valence DMRG-CASPT2 Study. Bull. Chem. Soc. Jpn. 2014, 87, 1071-1073.

(76) Shirai, S.; Kurashige, Y.; Yanai, T. Computational Evidence of Inversion of $1 \mathrm{La}$ and $1 \mathrm{Lb}$-Derived Excited States in Naphthalene Excimer Formation from ab Initio Multireference Theory with Large Active Space: DMRG-CASPT2 Study. J. Chem. Theory Comput. 2016, 12, 2366-2372.

(77) Kutzelnigg, W.; Mukherjee, D. Cumulant expansion of the reduced density matrices. J. Chem. Phys. 1999, 110, 2800-2809.

(78) Kutzelnigg, W.; Mukherjee, D. Normal order and extended Wick theorem for a multiconfiguration reference wave function. $J$. Chem. Phys. 1997, 107, 432-449.

(79) Zgid, D.; Ghosh, D.; Neuscamman, E.; Chan, G. K.-L. A study of cumulant approximations to n-electron valence multireference perturbation theory. J. Chem. Phys. 2009, 130, 194107.

(80) Saitow, M.; Kurashige, Y.; Yanai, T. Multireference configuration interaction theory using cumulant reconstruction with internal contraction of density matrix renormalization group wave function. $J$. Chem. Phys. 2013, 139, 044118.

(81) Neuscamman, E.; Yanai, T.; Chan, G. K.-L. A review of canonical transformation theory. Int. Rev. Phys. Chem. 2010, 29, 231271.

(82) Zgid, D.; Nooijen, M. Obtaining the two-body density matrix in the density matrix renormalization group method. J. Chem. Phys. 2008, $128,144115$.

(83) Sharma, S.; Chan, G. K.-L. Spin-adapted density matrix renormalization group algorithms for quantum chemistry. J. Chem. Phys. 2012, 136, 124121.

(84) BLOCK verion 1.1: An implementation of the density matrix renormalization group (DMRG) algorithm for quantum chemistry. https://github.com/sanshar/Block.

(85) Guo, S.; Watson, M. A.; Hu, W.; Sun, Q.; Chan, G. K.-L. NElectron Valence State Perturbation Theory Based on a Density Matrix Renormalization Group Reference Function, with Applications to the Chromium Dimer and a Trimer Model of Poly(p-Phenylenevinylene). J. Chem. Theory Comput. 2016, 12, 1583-1591.
(86) Sayfutyarova, E. R.; Chan, G. K.-L. A state interaction spin-orbit coupling density matrix renormalization group method. J. Chem. Phys. 2016, 144, 234301.

(87) Nakatani, N.; Guo, S. Density matrix renormalization group (DMRG) method as a common tool for large active-space CASSCF/ CASPT2 calculations. J. Chem. Phys. 2017, 146, 094102.

(88) Meyer, W. In Methods of Electronic Structure Theory; Schaefer, H. F., Ed.; Springer US: Boston, MA, 1977; pp 413-446.

(89) Sharma, S.; Chan, G. K.-L. Communication: A flexible multireference perturbation theory by minimizing the Hylleraas functional with matrix product states. J. Chem. Phys. 2014, 141, 111101.

(90) Sokolov, A. Y.; Chan, G. K.-L. A time-dependent formulation of multi-reference perturbation theory. J. Chem. Phys. 2016, 144, 064102.

(91) Sokolov, A. Y.; Guo, S.; Ronca, E.; Chan, G. K.-L. Timedependent $\mathrm{N}$-electron valence perturbation theory with matrix product state reference wavefunctions for large active spaces and basis sets: Applications to the chromium dimer and all-trans polyenes. J. Chem. Phys. 2017, 146, 244102.

(92) Roemelt, M.; Guo, S.; Chan, G. K.-L. A projected approximation to strongly contracted $\mathrm{N}$-electron valence perturbation theory for DMRG wavefunctions. J. Chem. Phys. 2016, 144, 204113.

(93) Sharma, S.; Jeanmairet, G.; Alavi, A. Quasi-degenerate perturbation theory using matrix product states. J. Chem. Phys. 2016, 144, 034103.

(94) Vahtras, O.; Almlöf, J.; Feyereisen, M. Integral approximations for LCAO-SCF calculations. Chem. Phys. Lett. 1993, 213, 514-518.

(95) Nieplocha, J.; Palmer, B.; Tipparaju, V.; Krishnan, M.; Trease, H.; Aprà, E. Advances, Applications and Performance of the Global Arrays Shared Memory Programming Toolkit. Int. J. High Perform. Comput. Appl. 2006, 20, 203-231.

(96) Dinan, J.; Balaji, P.; Hammond, J. R.; Krishnamoorthy, S.; Tipparaju, V. Supporting the Global Arrays PGAS Model Using MPI One-Sided Communication. 2012 IEEE 26th International Parallel and Distributed Processing Symposium. 2012; pp 739-750.

(97) The $\mathrm{cu}(4)$ approximated 4-TRDM is obtained by making direct use of the cumulant expansion $D_{\text {ghij }}^{k l m n} \approx 16 D_{g h i}^{k l m} \wedge D_{j}^{n}+18 D_{g h}^{k l} \wedge D_{i j}^{m n}-$ $144 D_{g h}^{k l} \wedge D_{i}^{m} \wedge D_{j}^{n}+96 D_{g}^{l} \wedge D_{h}^{k} \wedge D_{i}^{m} \wedge D_{j}^{n}$ (see also ref 59) where 1-, 2-, and 3-TRDMs are used for $D_{g}^{k}, D_{g h}^{k l}$ and $D_{g h i}^{k l m}$, respectively.

(98) Kendall, R. A., Jr.; Dunning, T. H.; Harrison, R. J. Electron affinities of the first-row atoms revisited. Systematic basis sets and wave functions. J. Chem. Phys. 1992, 96, 6796-6806.

(99) Irie, M.; Fukaminato, T.; Matsuda, K.; Kobatake, S. Photochromism of Diarylethene Molecules and Crystals: Memories, Switches, and Actuators. Chem. Rev. 2014, 114, 12174-12277.

(100) Irie, M.; Lifka, T.; Kobatake, S.; Kato, N. Photochromism of 1,2-Bis (2-methyl-5-phenyl-3-thienyl)perfluorocyclopentene in a Single-Crystalline Phase. J. Am. Chem. Soc. 2000, 122, 4871-4876.

(101) Uchida, K.; Matsuoka, T.; Kobatake, S.; Yamaguchi, T.; Irie, M. Substituent effect on the photochromic reactivity of bis(2-thienyl)perfluorocyclopentenes. Tetrahedron 2001, 57, 4559-4565.

(102) Becke, A. D. Density-functional thermochemistry. III. The role of exact exchange. J. Chem. Phys. 1993, 98, 5648-5652.

(103) Grimme, S.; Antony, J.; Ehrlich, S.; Krieg, H. A consistent and accurate $a b$ initio parametrization of density functional dispersion correction (DFT-D) for the 94 elements H-Pu. J. Chem. Phys. 2010, 132, 154104.

(104) Schäfer, A.; Huber, C.; Ahlrichs, R. Fully optimized contracted Gaussian basis sets of triple zeta valence quality for atoms $\mathrm{Li}$ to $\mathrm{Kr}$. J. Chem. Phys. 1994, 100, 5829-5835.

(105) Schäfer, A.; Horn, H.; Ahlrichs, R. Fully optimized contracted Gaussian basis sets for atoms Li to Kr. J. Chem. Phys. 1992, 97, 25712577.

(106) Forsberg, N.; Malmqvist, P.-Å. Multiconfiguration perturbation theory with imaginary level shift. Chem. Phys. Lett. 1997, 274, 196204.

(107) Ghigo, G.; Roos, B. O.; Malmqvist, P.-Å. A modified definition of the zeroth-order Hamiltonian in multiconfigurational perturbation theory (CASPT2). Chem. Phys. Lett. 2004, 396, 142-149. 
(108) Piard, J.; Ishibashi, Y.; Saito, H.; Métivier, R.; Nakatani, K.; Gavrel, G.; Yu, P.; Miyasaka, H. Multiphoton-gated cycloreversion reaction of a photochromic 1,2-bis(thiazolyl) perfluorocyclopentene diarylethene derivative. J. Photochem. Photobiol., A 2012, 234, 57-65. In honour of Monique M. Martin.

(109) Roos, B. O.; Andersson, K. Multiconfigurational perturbation theory with level shift - the $\mathrm{Cr} 2$ potential revisited. Chem. Phys. Lett. 1995, 245, 215-223. 